\title{
Diskontierung durch Replikation
}

Richtiges und Falsches bei der Unternehmensbewertung

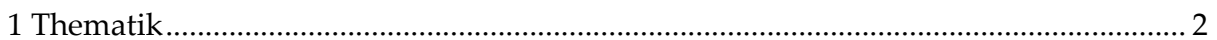

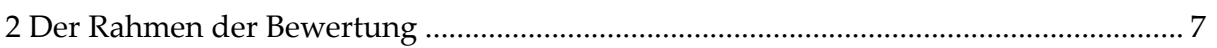

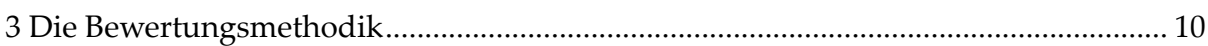

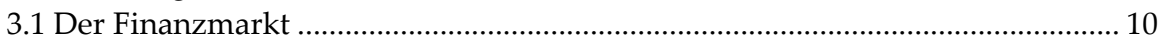

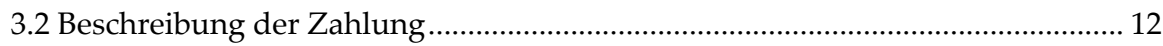

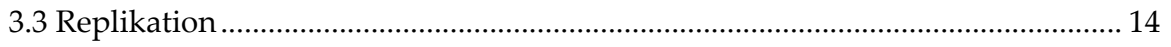

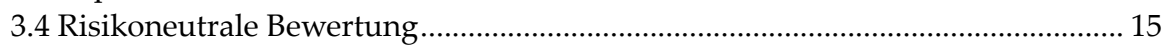

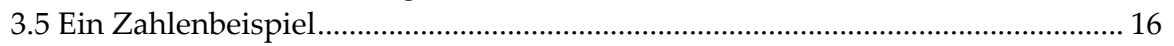

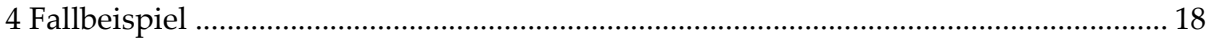

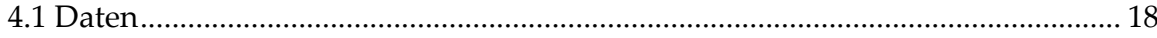

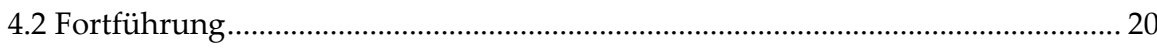

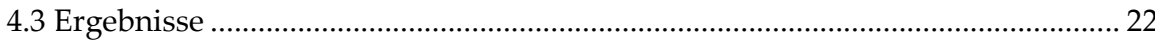

\section{Thematik}

Eine weite Klasse von Modellen bewertet eine Unternehmung anhand der Erträge, Überschüsse oder Zahlungen, die sie in Zukunft für die Berechtigten generiert. Je nach Modell haben die erzeugten Zahlungen die Bedeutung von Dividenden, Gewinnen, Freien Cashflows oder von Excess Returns, weshalb jede Bewertung als Erstes eine Wahl des Ansatzes voraussetzt. Hier bieten sich das Dividenden-Wachstums-Modell, eine Ertragsbewertung, der DCF-Ansatz oder eine Bewertung anhand der Residualeinkommen. Wenn der Ansatz gewählt und die Zahlungen der Art nach spezifiziert sind, wird als Zweites eine Vorschau der Zahlungen für alle kommenden Jahre erstellt. Selbstverständlich muss diese Vorschau auf geeignete Weise die mutmaßliche Höhen und die jeweiligen Unsicherheiten der zukünftigen Zahlungen erfassen sowie ihre gegenseitigen stochastischen Abhängigkeiten. Hinweise dafür bieten die Ge- 
schäftsplanung der betreffenden Unternehmung und die Prognose der allgemeinen Wirtschaftsentwicklung, von der die Unternehmung abhängig ist. Diese Planungen und Prognosen münden in eine Folge unsicherer Zahlungen, $\widetilde{Z}_{1}, \widetilde{Z}_{2}, \widetilde{Z}_{3}, \ldots$, deren Wahrscheinlichkeitsverteilungen typischerweise von der allgemeinen Wirtschaftsentwicklung abhängen. Im dritten Schritt der Bewertung muss der Wert der unsicheren Zahlungsreihe $\widetilde{Z}_{1}, \widetilde{Z}_{2}, \widetilde{Z}_{3}, \ldots$ ermittelt werden. ${ }^{1}$ Für diesen dritten Schritt wird oft eine "vereinfachte Diskontierung" (simplified discounting rule) verwendet. Hierbei werden die unsicheren Zahlungen $\tilde{Z}_{t}$ der kommenden Jahre durch ihre Erwartungswerte $E\left[\tilde{Z}_{t}\right]$ beschrieben, $t=1,2,3, \ldots$, und die Erwartungswerte werden sodann mit einem Satz $r$ diskontiert, der neben dem Zinssatz eine Risikoprämie beinhaltet. Für die Bestimmung des Diskontsatzes $r$ wird vielfach das CAPM vorgeschlagen und dazu versucht, das Beta zu bestimmen. Auch gibt es eine umfassende Theorie, wie eine Fremdfinanzierung und wie Unternehmenssteuern berücksichtigt werden können. Diese Theorie führt auf die Weighted-Average-Cost-of-Capital und die Miles-Ezzell-Cost-ofCapital. Jedenfalls wird bei der vereinfachten Diskontierung

$$
W=\frac{E\left[\widetilde{Z}_{1}\right]}{1+r}+\frac{E\left[\widetilde{Z}_{2}\right]}{(1+r)^{2}}+\frac{E\left[\widetilde{Z}_{3}\right]}{(1+r)^{3}}+\ldots
$$

als "Wert" der Zahlungsreihe $\widetilde{Z}_{1}, \widetilde{Z}_{2}, \widetilde{Z}_{3}, \ldots$ und deshalb als Wert der Unternehmung angesehen. Hinter der Wertformel (1) stehen vier Problemkreise, die teils schon länger bekannt sind, oder deren man sich in den letzten Jahren bewußter geworden ist.

Der erste Problemkreis betrifft die Frage, ob der Wert einer Unternehmung in der modernen Gesellschaft mit verschiedenen Anspruchsgruppen und den Wünschen nach sozialer Gerechtigkeit mit Erhalt von Arbeitsplätzen, nach dem Respektieren ethischer Grundhaltungen und dem Verlangen von Schutz der Umwelt sich allein aus den Zahlungsüberschüssen zugunsten von Finanzinvestoren ergibt. Es wird argumentiert, dass die Unternehmung zahlreiche implizite Kontrakte mit "Stakeholdern" zu erfüllen habe und dass ihrer Führung besondere Verantwortung für die Gesellschaft und die Zukunft zukomme. Es wird im gesellschaftlichen Leben "wertbestimmende Faktoren" geben, die sich nicht in den von der Unternehmung für Kapitalgeber generierten Zahlungen ausdrücken. Hier dürfte die Antwort auch nicht überall die selbe sein. Jede Gesellschaft hat aufgrund ihrer Traditionen und ihrer Bedürfnisse eigene Vorstellungen von der Unternehmung entwickelt, die in den Kulturkreisen unterschiedlich akzentuiert sind. Auf diese erste Thematik treten wir aber nicht ein.

Beim zweiten Problemkreis wird die finanzwirtschaftliche Perspektive angenommen und der Arbeitshypothese gefolgt, dass sich der Unternehmenswert allein aus den

1 Nach dem Dividend Discount Model ist der Wert der Unternehmung gleich dem Wert der ausgeschütteten Zahlungsüberschüsse, sofern die Transversalität gilt, so dass allfällige Restwerte, Lasten oder Perlen bei unendlichen Horizont keinen Einfluß mehr haben. 
Zahlungen ableitet, die sie für die Berechtigten generiert. Doch es wird die Abhängigkeit dieser Zahlungen von den beteiligten und handelnden Personen thematisiert, die unterschiedlich informiert und motiviert sind. Zu diesen Personen gehören die Eigenkapitalgeber - sind es Großinvestoren oder Publikumsaktionäre? - sowie Fremdkapitalgeber, vor allem Banken. Allein die Zusammensetzung der Kapitalgeber hat Einfluß auf die Wirtschaftstätigkeit. Weiter sind die Manager in der Unternehmung verschiedensten Motivationen ausgesetzt und verfügen bei ihren Entscheidungen über einen erheblichen "diskretionären" Freiraum. Es kommen Aufsichtsgremien und Regulierungen hinzu, eventuell die Einflußnahme der Politik. Eine umfangreiche Literatur hat auf diese Einflüsse hingewiesen und sie modelliert, vor allem durch Prinzipal-Agenten-Modelle. Im Ergebnis kann ein Unternehmen nicht "an sich" bewertet werden sondern immer nur im Hinblick auf eine gewisse Eigentümerstruktur sowie eine Corporate Governance, sprich, eine gewisse Finanzierung, weil dadurch die Geschäfts- und Investitionspläne beeinflußt sind. Die generierten Überschüsse können nicht von diesem personellen Umfeld und von der Kapitalstruktur gelöst werden. ${ }^{2}$

Beim dritten Problemkreis geht es um die Wahl der bewertungsrelevanten Zahlungen. Sollen es Cashflows, Gewinne, Dividenden sein? Diese “Überschüsse" unterscheiden sich durch Investitionen, die neben den laufenden Geschäften berücksichtigt werden und daher in die Bewertung einfließen. Schon der Begriff des Freien Cashflows verdeutlicht die Frage, ob lediglich ein operativer Plan oder eine Kombination aus Geschäftstätigkeit und Investitionstätigkeit bewertet werden soll. Selbstverständlich gibt es Varianten für die budgetierten Investitionen, und es hängt von ihnen ab, wie hoch die Zahlungsüberschüsse letztlich sind. Dabei können komplexe Interaktionen auftreten, so weil die Unternehmung kaum einen starren Investitionsplan folgen sondern in Abhängigkeit der realisierten Ergebnisse investieren wird. Die Unternehmung kann Realoptionen haben und, spätere Informationen berücksichtigend, ihre Investitionen größer oder geringer auslegen. Dadurch verändern sich die nach Investition verbleibenden Zahlungsüberschüsse nicht nur der Höhe nach sondern auch in ihren stochastischen Eigenschaften. $^{\mathbf{3}}$

Wir wollen uns im Folgenden dem vierten Problemkreis der Unternehmensbewertung zuwenden. Die Frage lautet: Darf, bei einer rein finanztheoretischen Sicht, die gemäß (1) bestimmte Größe $W$ als Wert der Zahlungsreihe $\widetilde{Z}_{1}, \widetilde{Z}_{2}, \widetilde{Z}_{3}, \ldots$ angesehen werden? Den Wert einer zukünftigen und daher meistens unsicheren Zahlung oder einer sich in die Zukunft erstreckenden stochastischen Zahlungsreihe zu bestimmen, heißt, zu diskontieren. Die Frage also lautet, ob die in (1) angegebene Formel korrekt die Diskontierung beschreibt.

2

Unter vereinfachenden Annahmen kommt dann derselbe Wert heraus - MODIGLIANI und MILLER konnten Irrelevanz-Thesen aufstellen. Doch in feineren Modellierungen der Realität, insbesondere bei Berücksichtigung von Realoptionen, haben die Investitionsentscheidungen und die Finanzierung Einfluß auf den Unternehmenswert. 
Über diese Frage hat in der jüngeren Forschung eine intensive und teils kontroverse Diskussion eingesetzt. Dabei geht es nicht um die vielleicht praktische Schwierigkeit, die Diskontrate $r$ zu ermitteln, und dazu im CAPM das Beta korrekt zu bestimmen. Der Punkt ist auch nicht, ob $r$ den Weighted-Average-Cost-of-Capital oder den MilesEzzell-Cost-of-Capital entsprechen soll. Es geht um die prinzipielle Korrektheit der sogenannten Risikoprämienmethode. Bei ihr wird

$$
E\left[\widetilde{Z}_{t}\right] /(1+r)^{t}
$$

als Barwert einer zu $t$ fälligen, unsicheren Zahlung $\widetilde{Z}_{t}$ angesehen. Während im Zähler von (2) der Erwartungswert des unsicheren Zahlungsüberschusses steht, wird das Risiko dadurch berücksichtigt, dass im Nenner $r$ sich aus dem Zinssatz und einem Zuschlag zusammensetzt, einer Risikoprämie. ${ }^{4}$ Noch dazu ist in (1) die Diskontrate für alle Jahre, zu denen Zahlungsüberschüsse anfallen, gleich. Verschiedene Teilfragen sind aufgeworfen worden. ${ }^{5}$

- So ging es ursprünglich es um eine Klärung, wie das CAPM zur Bestimmung der Diskontfaktoren bei der Risikoprämienmethode (2) von einem auf mehrere Jahre übertragen werden kann, vergleiche BRENNAN $(1973,1979)$.

- Im Kontext mehrerer Jahre gewinnen Verteilungsparameter wie der Erwartungswert in (2) neue Bedeutung, weil die Wahrscheinlichkeitsverteilung im zunehmendem $t$ typischerweise immer stärkere Rechtsschiefe zeigt (FAMA 1977).

- Drittens hat BALLWIESER postuliert, dass die Diskontrate $r$ in (1) vielleicht nicht für alle Jahre dieselbe sein darf sondern tendenziell für Zahlungen mit späteren Fälligkeiten geringer werden müßte, weil sie ansonsten zu stark diskontiert würden. ${ }^{6}$

- Viertens wurde gefragt, ob anstelle der Risikoprämienmethode (2) nicht eine Diskontierung vorzunehmen sei, bei der im Zähler anstelle des Erwartungswerts ein Sicherheitsäquivalent stehen müsse - Berücksichtigung des Risikos im Zähler durch einen Risikoabschlag - während im Nenner der Zinssatz als Rendite der risikofreien Anlage zu stehen habe.

- Fünftens wurde mit dem Begriff der "Risikoauflösung" thematisiert, welche Informationen über die Verteilung von $\widetilde{Z}_{t}$ im Verlauf der Jahre $1,2, \ldots, t-1$ eintreten und in wie weit sich dadurch ein Einfluß auf den Wert der Zahlung ergibt.

- Schließlich wurde untersucht, was sich ändert, wenn die Wirtschaftsentwicklung, vertreten durch einen Index, nicht die klassische Voraussetzung seriell unkorrelierter Renditen erfüllt und wenn der Zinssatz stochastisch schwankt. ${ }^{7}$

4 Hierzu: BlACK 1988, BHATTACHARYA und CONSTANTINIDES (1988) und RiCHTER (2001).

5 Siehe KRUSCHWitZ / LÖFFLER (1998). SCHWETZLER (2000), KRUSCHWITZ (2002), KÜRSTEN (2002), WILHELM (2002).

6 BALLWIESER (1990), p.171. 
Im Folgenden möchte ich zu einigen dieser Teilfragen Stellung nehmen. Dazu wird eine Methode eingesetzt, die ab 1970 entwickelt wurde als die generelle Bewertungsmethode für "financial claims" akzeptiert ist. Es ist die Replikation der zu bewertenden Zahlung in einem arbitragefreien Finanzmarkt. ${ }^{8}$ Dabei wird die zu diskontierende oder zu bewertende Zahlung durch eine geeignete Kombination von Finanzinstrumenten nachgebildet - etwa einer Geldanlage zum Zinssatz und einer Investitionen in einen Index. Da die Investoren im Finanzmarkt nur auf die Zahlungen, ihre Fälligkeiten, ihre erwarteten Höhen und ihre stochastischen Eigenschaften achten, hat der von der Unternehmung erzeugte Zahlungsüberschuß denselben Wert wie das Portfolio, das ihn in seiner Fälligkeit, seiner erwarteten Höhe und seinen stochastischen Eigenschaften nachbildet. Da die Preise der Instrumente in diesem "Replikationsportfolio" vorliegen, ist durch die Zusammensetzung dieses Portfolios der Wert des zu diskontierenden Zahlungsüberschusses ermittelt. ${ }^{9}$ Vom Grundsatz her ist dieser Ansatz bekannt, doch er wird teils recht abstrakt vorgetragen. Wir wollen im Folgenden sehen, dass sich die Diskontierung durch Replikation einfach anwenden und praxisnah gestalten läßt. Auch dieses Ziel wird mit der nachstehenden Darstellung verfolgt.

Es ist noch nachzutragen, was unter dem Wert einer unsicheren Zahlung verstanden wird: Es ist der Preis, den diese Zahlung in einem gut funktionierenden Finanzmarkt hat. Ein gut funktionierender Finanzmarkt soll vor allem arbitragefrei sein. Das heißt, es lassen sich Portfolios nicht so umstrukturieren, dass - ohne weiteres Risiko - ein zusätzlicher Gewinn oder ein "Free Lunch" möglich ist. Im Grunde heißt dies: Der Finanzmarkt soll sich im Gleichgewicht befinden. Sodann soll der Finanzmarkt so gut entwickelt sein und so viele Instrumente bieten, dass sich die von der Unternehmung erzeugten Überschüsse, die bewertet werden sollen, mit Kombinationen der gehandelten Finanzinstrumente replizieren lassen. Das ist eine Annahme hinsichtlich der Vollständigkeit des Marktes. Drittens soll der Finanzmarkt so groß sein, dass das "Hineinbringen" der zu bewertenden Zahlung keine Rückwirkung auf die Preisbildung der vorhandenen Finanzinstrumente im Markt hat. Er soll also dem in der Mikroökonomie üblichen "atomistischen" Bild genügen.

Die Vorstellung, dass der Wert gleich dem Preis in einem gut funktionierenden Markt ist, wurde der allgemeinen Gleichgewichtstheorie entlehnt, die um 1970 vor allem von ARROW und DeBreU entwickelt wurde. Damit soll die tiefe Auseinandersetzung um diesen in der Wirtschaft wichtigen Begriff des Werts nicht außer Kraft gesetzt

7 LAITENBERGER (2004).

8 In dieser Denkrichtung folgten die Arbitrage Pricing Theory (Ross 1976) und weitere Arbeiten, so von RUBINSTEIN (1976), BRENNAN (1979), HARRISON und KREPS (1979).

9 Um ein einfaches Beispiel zu nennen: Eine Firma produziert Orangensaft. Im Markt gibt es einen gut funktionierenden Handel mit Konzentrat, Zucker und Wasser, und aus diesen Ingredienzen kann durch Mischung ein Getränk erzeugt werden, dass in allem für die Konsumenten relevanten Eigenschaften mit dem produzierten Saft übereinstimmt. Dann muss im gut funktionierenden Markt der Preis für den erzeugten Orangensaft mit den Preisen der sie nachbildenden Ingredienzen übereinstimmen. 
werden. ${ }^{10}$ In der Tradition von CARL MENGER (1840-1921), Begründer der österreichischen Schule, wird der Wert eines Objekts vielfach mit dem Nutzen assoziiert, den es stiftet. In der Tat hat die Gleichgewichtstheorie oder die Mikroökonomie den Zusammenhang zwischen dem Preis (im Marktgleichgewicht) und dem Nutzen eines Individuums aufgezeigt: Für jeden Einzelnen ist der Grenznutzen eines Objekts (bei einer Variation der Quantität) gleich dem Preis. Es gibt Bewertungsmodelle für Unternehmen, die von der Risikonutzenfunktion eines Investors ausgehen. Sie rücken das Sicherheitsäquivalent eines unsicheren Ergebnisses in den Mittelpunkt. Allerdings ist dies zu beachten:

1. Für einen Entscheidungsträger ist das Sicherheitsäquivalent jener Geldbetrag, der denselben Erwartungsnutzen aufweist wie das unsichere Ergebnis. Beim Zusammenhang zwischen Preis und Nutzen wird indessen die Bedeutung des Grenznutzens hervorgehoben. Der Preis ist für alle Marktteilnehmer gleich dem Grenznutzen, der mit einer Variation der Quantität verbunden ist. Von daher dürfen Bewertungsmodelle, die auf das Sicherheitsäquivalent eines Investors abstellen, nur mit entsprechenden Korrekturen übernommen werden.

2. In einem Finanzmarkt sind nur "systematische" Risiken mit einer Prämie verbunden, während "unsystematische Risiken" diversifizierbar sind und daher bei einer Bewertung im Markt einen Preis in Höhe ihres Erwartungswerts haben. Im Unterschied dazu hat ein einzelner Entscheidungsträger für jedes Risiko, das er übernimmt, einen Nutzenentgang, ungeachtet der Frage, ob es im Prinzip diversifizierbar wäre oder nicht.

Deshalb müssen Bewertungsmodelle, die auf das Sicherheitsäquivalent abheben, mit entsprechender Vorsicht verwendet werden. Ein Bewertung anhand des Sicherheitsäquivalents eines Investors muss zwar nicht falsch sein, sie kann durchaus für gewisse Spezifikationen den korrekten Wert liefern. Jedoch zeigen Beispiele, dass sie das nur unter gewissen Voraussetzungen tun. Gleiches gilt auch für die Risikoprämienmethode. Hier hat eine Untersuchung jener Bedingungen eingesetzt, unter denen sie mit der im Finance generell üblichen Bewertung durch Replikation konsistent sind.

\section{$2 \quad$ Der Rahmen der Bewertung}

Eine zu bewertende Zahlung kann nicht vom Hintergrund des Finanzmarktes gelöst werden, denn es wird mit ihrem Wert jener Preis gesucht, den sie im zugrunde gelegten Markt hätte. Nun gibt es in jedem Finanzmarkt Risiken, die noch diversifiziert werden können und solche, die nicht diversifizierbar sind. Für diese Unterscheidung

10 Zum Beispiel: HICKS (1939), STÜTZEL (1952, 1972), WITTMANN (1956). 
ist bereits ein Modell verlangt, etwa das CAPM oder die Arbitrage Pricing Theory (APT). Die diversifizierbaren Risiken werden im CAPM als unsystematisch und in der APT als spezifisch bezeichnet. Da die diversifizierbaren Risiken im Markt zum Ausgleich gebracht werden können, spielen sie für die Preisbildung (und damit die Bewertung) keine Rolle mehr. Die unsystematische oder spezifische Unsicherheit in einem Zahlungsüberschuß wird daher so bewertet wie eine sichere Zahlung in Höhe ihres Erwartungswerts. Dies ist wichtig, weil oft in Beispielen risikobehaftete Zahlungsüberschüsse betrachtet werden, oft als "Lotterie" bezeichnet, deren Risiko durch Diversifikation im Markt zum Verschwinden gebracht werden könnte. Damit würde eine solche Lotterie im Markt anhand ihres erwarteten Ergebnisses bepreist, und ihr heutiger Wert entspricht daher dem diskontierten Erwartungswert, der wie eine sichere Zahlung mit dem Zinssatz diskontiert wird. ${ }^{11}$

Der Punkt bei der Bewertung unsicherer Zahlungsüberschüsse entsteht also insoweit, als diese nicht-diversifizierbare Risiken enthalten. Denn die Investoren im Markt sind risikoavers, weshalb sich bei der Preisbildung nicht diversifizierbarer Risiken Prämien bilden. Die Marktprämien für nicht-diversifizierbare Risiken werden bei der Bewertung stets hineinspielen, wenn der von einer Unternehmung erzeugte Zahlungsüberschuß nicht-diversifizierbare Risiken aufweist - vielleicht neben diversifizierbaren Risikoanteilen.

Bei der Beschreibung eines Zahlungsüberschusses genügt es daher nicht, auf dessen Wahrscheinlichkeitsverteilung einzugehen, vielleicht auf die Standardabweichung (neben dem Erwartungswert der zu diskontierenden Zahlung). Es muss gesagt werden, in welcher (stochastischen) Beziehung der zu bewertende Überschuß zu anderen, nicht-diversifizierbaren Risiken der im Markt gehandelten Instrumente steht. Wird das CAPM zugrunde gelegt, werden nicht diversifizierbare Risiken durch ein einziges Instrument perfekt beschrieben: das Marktportfolio. In diesem Fall muss für den zu diskontierenden Zahlungsüberschuß bekannt sein, wie er mit dem Index, der das Marktportfolio repräsentiert, variiert. Unter Umständen genügt dazu die Kenntnis von Standardabweichung und Korrelation. Wird die APT zugrunde gelegt, könnte es durchaus mehr als einen Faktor geben, der mit einer Prämie verbunden ist. Dann müssen die "Exposures" bekannt sein, die der zu diskontierende Überschuß hinsichtlich aller Faktoren aufweist.

Im Folgenden beschreiben wir den Finanzmarkt (im Sinne der APT) durch ein EinFaktor-Modell. ${ }^{12}$ Mit einem Ein-Faktor-Modell wird zugegebenermaßen eine Modellbildung des Finanzmarktes vorgenommen. Es wird studiert, welchen Preis der Zah-

11 Das ist kompliziert genug, wenn es keinen zum Bewertungszeitpunkt bekannten Zinssatz für die entsprechende Fälligkeit der Zahlung gibt. Wir gehen hier davon aus, dass die Zinssätze für alle Fälligkeiten gegeben sind.

12 Wir vermeiden dabei, das Ein-Faktor-Modell als CAPM anzusprechen, weil der Begriff des CAPM an die unzähligen empirischen Studien erinnert, in denen kontrovers diskutiert wurde, mit welcher Genauigkeit "Beta" die wirklichen Finanzmärkte zu beschreiben vermag. 
lungsüberschuß in diesem Modell eines Finanzmarktes hätte. ${ }^{13}$ Es soll einen einzigen Index geben, und eine Kapitalanlage in diesen Index ist mit einer Prämie verbunden. Risiken, die nicht mit dem Index korreliert sind, werden als diversifizierbar betrachtet.

Die Beschreibung des Zahlungsüberschusses läuft daher vor allem auf die Beschreibung der Abhängigkeit hinaus, in der die Zahlung zu den Werten des Index steht, den dieser zum Zeitpunkt der Fälligkeit des Zahlungsüberschusses hat. Dabei darf die Entwicklung des Indexes als die der "allgemeinen Wirtschaft" interpretiert werden. Somit wird für die Beschreibung des zu bewertenden Zahlungsüberschusses untersucht, wie er von der "allgemeinen Wirtschaftsentwicklung" abhängt. Die grafische Darstellung der Abhängigkeit des Zahlungsüberschusses vom Index ist das PayoffDiagramm. Da der Index das nicht-diversifizierbare Risiko und damit die Risikoprämie verkörpert, ist allein die Abhängigkeit vom Index relevant für den Wert. Der Payoff enthält daher die wertrelevanten Informationen.

Zur Bewertung allgemeiner Payoffs wurde um 1970 die Idee der Replikation entwikkelt, bei der ein zu bewertender Payoff durch ein Portfolio anderer Instrumente nachgebildet wird, deren Preise im Finanzmarkt bekannt sind. Wenn ein geeignet zusammengesetztes Portfolio denselben Payoff besitzt wie ein zu bewertendes Instrument, dann sind das Portfolio und das Instrument in allen Aspekten, die im Finanzmarkt den Wert bestimmen, identisch. Folglich muss der Wert des Instruments mit der Summe der Werte der Komponenten des Portfolios übereinstimmen. ${ }^{14}$ Diese Bewertungsmethode wurde ursprünglich für Optionen gedacht. Optionen werden als bedingte Zahlungen (contingent claims) aufgefaßt, wobei die Höhe der Zahlung, die der Inhaber bei Fälligkeit erhält, eine Funktion des Kurses ist, den das Underlying dann haben wird. Nicht notwendig muss es sich beim Underlying um den Index handeln.

Genau so können die Zahlungsüberschüsse, die eine Unternehmung generiert, als contingent claims aufgefaßt werden. Gewinne oder Cashflows einer Firma sind nicht vom allgemeinen Marktgeschehen unabhängig. Vielmehr sind sie in der Regel höher, wenn die allgemeine Wirtschaftsentwicklung besser läuft, und sie sind geringer, wenn die Entwicklung der wirtschaftlichen Umgebung schwächer ist. Die "allgemeine Wirtschaftsentwicklung" als Underlying denkt man sich als durch einen Marktindex repräsentiert und quantifiziert. So werden die von der Unternehmung generierten Zahlungsüberschüsse vor allem eine Funktion des Index. Zusätzlich können die von einer Unternehmung generierten Zahlungen unsystematische oder firmenspezifische Zufälligkeiten aufweisen, die wie gesagt im Finanzmarkt keine Relevanz für den Wert haben.

13 Eine jede Bewertung geht von einem Finanzmarkt-Modell aus, und der in einem konkreten Umfeld für eine Transaktion ausgehandelte Preis kann sich durchaus von diesem Wert unterscheiden.

14 Brennan (1979), Harrison / Kreps (1979), Hubermann (1982); für eine Einführung und die frühe Literatur siehe Spremann (1986). 
Anders als im Fall von Optionen dürfte die Abhängigkeit der systematischen Zahlungen einer Unternehmung vom Markgeschehen sogar linear sein, weil vielfach die Unternehmung ein konstantes Exposure gegenüber der Wirtschaftsentwicklung zeigt (und das wäre nur anders, wenn Realoptionen zu modellieren sind). Aufgrund der Linearität genügen sehr einfache Diskretisierungen der Wirtschaftsentwicklung (ein Binomialmodell muss nur eine einzige Verzweigung haben), um die Payoffs exakt zu beschreiben. Folglich genügen, wenn Gewinne oder Cashflows einer Firma in der Vorschau aufgestellt werden, ausgesprochen einfache Beschreibungen der zu bewertenden Zahlungen. Zwar ist es nicht hinreichend, nur ihren Erwartungswert oder Median zu kennen. Doch genügen bereits zwei Realisationen der zukünftigen Gewinne oder Cashflows, um die Bewertung durchführen zu können. Weil eine dermaßen einfache Beschreibung der Zahlungen genügt, sind die nachstehenden Ausführungen recht praxisnah. Wir führen nun diesen Bewertungsansatz vor (Teil 3) und illustrieren sie anhand eines Fallbeispiels (Teil 4).

\section{$3 \quad$ Die Bewertungsmethodik}

\subsection{Der Finanzmarkt}

Gesucht ist der Wert $W$, den die unsichere Folge von Zahlungen $\widetilde{Z}_{1}, \widetilde{Z}_{2}, \widetilde{Z}_{3}, \ldots$ im Finanzmarkt besitzt. Hierzu treffen wir fünf Annahmen. Die ersten drei Annahmen beschreiben den Finanzmarkt, darunter den Index, der die "allgemeine Wirtschaftsentwicklung" beschreibt. Die vierte Annahme beschreibt die von der Unternehmung generierten Zahlungen in Bezug auf diesen Finanzmarkt.

1. Annahme: Der "Wert" wird als Preis verstanden, den der Zahlungsstrom im Finanzmarkt erzielen würde. Annahme: Der Finanzmarkt ist arbitragefrei und enthält hinreichend viele Instrumente Kontrakte, so dass die Wertadditivität gilt: Folgerungen: Die Zahlungen des Zahlungsstroms $\widetilde{Z}_{1}, \widetilde{Z}_{2}, \widetilde{Z}_{3}, \ldots$ können im Finanzmarkt getrennt gekauft und verkauft werden. Der Wert $W$ des Zahlungsstroms $\widetilde{Z}_{1}, \widetilde{Z}_{2}, \widetilde{Z}_{3}, \ldots$ ist gleich der Summe der Werte $W_{t}\left(\widetilde{Z}_{t}\right)$ der einzelnen unsicheren Zahlungen $\widetilde{Z}_{t}, t=1,2, \ldots$ Die Aufgabe, den Wert der unsicheren Folge von Zahlungen $\widetilde{Z}_{1}, \widetilde{Z}_{2}, \widetilde{Z}_{3}, \ldots$ zu bestimmen, zerfällt somit in die Teilaufgaben, den Wert $W_{t}\left(\widetilde{Z}_{t}\right)$ der einzelnen unsicheren Zahlung $\widetilde{Z}_{t}, t=1,2, \ldots$ zu finden. 
2. Annahme: Es gibt einen Index, der das nicht diversifizierbare Risiko beschreibt. Die Rendite auf den Index erfülle die Standardannahmen zum Random Walk: Sie sei normalverteilt $-\mu$ sie der Erwartungswert und $\sigma$ die Standardabweichung der stetigen Rendite auf das dem Index zugrundeliegende Markt- oder Branchenportfolio. Diese Parameter sind gegeben. Zeitlich aufeinanderfolgende Renditen sind seriell unkorreliert. Die Höhe des Index $\widetilde{S}_{t}$ zum Zeitpunkt $t$ ist daher lognormalverteilt, während $\ln \widetilde{S}_{t}$ normalverteilt ist. Die zufällige Größe $\ln \widetilde{S}_{t}$ besitzt also den Erwartungswert $\ln S_{0}+t \cdot \mu$ und die Standardabweichung $\sqrt{t} \cdot \sigma$. Schätzungen beispielsweise für den Schweizer Aktienmarkt 1926-2003 sind $\hat{\mu}=7,61 \%$ und $\hat{\sigma}=19,01 \%$.

3. Annahme: Daneben soll es möglich sein, risikofrei Mittel anlegen und aufnehmen zu können. Der Zinssatz in stetiger Notation sei mit $i$ (wie interest) bezeichnet. Der Zinssatz sei für alle betrachteten Fälligkeiten $t$ bekannt (sicher). Für die Schweiz erscheint ein Zinssatz um 4\% realitätsnah.

Insoweit ist der Finanzmarkt beschrieben. Wir werden dem bei Wachstumsvorgängen generell üblichen Vorgehen folgen und die Logarithmen jener Größen betrachten, die dem Wachstumsvorgang unterliegen. Wenn sich der Index entsprechend

$$
\widetilde{S}_{t}=S_{0} \cdot\left(1+\widetilde{r}_{1}^{(e i n)}\right) \cdot\left(1+\widetilde{r}_{2}^{(e i n)}\right) \cdot \ldots \cdot\left(1+\widetilde{r}_{t}^{(e i n)}\right)
$$

entwickelt, $\widetilde{r}_{t}^{(e i n)}$ ist die einfache Rendite im Jahr $t$, dann ist sein Logarithmus

$$
\ln \widetilde{S}_{t}=\ln S_{0}+\ln \left(1+\widetilde{r}_{1}^{(e i n)}\right)+\ln \left(1+\widetilde{r}_{2}^{(e i n)}\right)+\ldots+\ln \left(1+\widetilde{r}_{t}^{(e i n)}\right)=\ln S_{0}+\widetilde{r}_{1}+\widetilde{r}_{2}+\ldots+\widetilde{r}_{t}
$$

die Summe von $\ln S_{0}$ und den Renditen $\widetilde{r}_{1}, \widetilde{r}_{2}, \ldots, \widetilde{r}_{t}$ in stetiger Schreibweise. Da die Renditen der einzelnen Perioden unabhängig voneinander sind, ist aufgrund des zentralen Grenzwertsatzes der Statistik der Logarithmus des Ergebnisses, $\ln \widetilde{S}_{t}$, normalverteilt. Diese für den Marktindex oft getroffenen Annahme gilt letztlich für viele Wachstumsvorgänge. So dürften auch die von der Unternehmung generierten Zahlungen lognormalverteilt sein, oder Verschiebungen der Zahlungsüberschüsse sind lognormalverteilt. ${ }^{15}$ Ihre Abhängigkeit vom Index sei durch die fünfte Annahme beschrieben:

Annahme 4: Die zu $t$ fällige Zahlung $\widetilde{Z}_{t}$ ist eine lineare Funktion des Marktindex $\widetilde{S}_{t}$, den dieser zu diesem Zeitpunkt haben wird, oder eben der Veränderung $\widetilde{S}_{t} / S_{0}$, die der Index vom Bewertungszeitpunkt bis zur Fälligkeit wird: $\widetilde{Z}_{t}=a_{t}+b_{t} \cdot\left(\widetilde{S}_{t} / S_{0}\right)+\widetilde{\varepsilon}_{t}$. Hier erfaßt $\widetilde{\varepsilon}_{t}$ unsystematische oder firmenspezifische Zufälligkeiten. Diese unsyste-

15

Wie bei der allgemeinen Wirtschaftsentwicklung (Index) macht sich auch bei den von der Unternehmung generierten Zahlungen eine gewisse Rechtsschiefe bemerkbar, die um so deutlicher ist, je weiter das Jahr $t$ in der Zukunft liegt. 
matischen Zufälligkeiten gleichen sich durch Diversifikation über verschiedene Unternehmen aus, weshalb $\widetilde{\varepsilon}_{t}$ keinen Einfluß auf den Wert ausübt. Um die Bezeichnungen nicht zu überladen, betrachten wir nur noch das systematische Risiko. Folglich unterstellen wir die Abhängigkeit der zu bewertenden Zahlung in der Form

$$
\tilde{Z}_{t}=a_{t}+b_{t} \cdot \tilde{S}_{t} / S_{0}=a_{t}+b_{t} \cdot\left(\tilde{r}_{1}+\tilde{r}_{2}+\ldots+\tilde{r}_{t}\right)
$$

Bemerkungen:

1. Hinsichtlich der Entstehung des Risikos von $\widetilde{Z}_{t}$ wird mit der Annahme 4 unterstellt, dass es von heute bis zur Fälligkeit $t$ heranreift, genau wie der Index zum Bewertungszeitpunkt 0 ist $S_{0}$ eine sichere Größe - bis $t$ zu $\widetilde{S}_{t}$ heranreift.

2. Die Annahme der vollen "Reifezeit" kann man modifizieren: Eine Variation von (5) besteht darin, für den zu diskontierenden Überschuß $\widetilde{Z}_{t}$ eine kürzere Reifezeit zu

unterstellen. So hieße $\widetilde{Z}_{t}=a_{t}+b_{t} \cdot\left(\widetilde{S}_{t} / \widetilde{S}_{t-1}\right)=a_{t}+b_{t} \cdot \widetilde{r}_{t}$, dass die im Jahr $t$ fällige Zahlung hinsichtlich ihres systematischen Risikos nur von der Wirtschaftsentwicklung (Index) im Jahr $t$ abhängt, nicht aber von der Indexentwicklung der Jahre $1,2, \ldots, t-1$. Allgemein könnte eine Reifezeit von $m$ Jahren ausgedrückt werden, indem (5) durch $\widetilde{Z}_{t}=a_{t}+b_{t} \cdot\left(\widetilde{S}_{t} / \widetilde{S}_{t-m}\right)=a_{t}+b_{t} \cdot\left(\widetilde{r}_{t-m+1}+\ldots+\widetilde{r}_{t}\right)$ ersetzt wird. Selbstverständlich übt die Länge der Reifezeit Einfluß auf den Wert der Zahlung $\tilde{Z}_{t}$ aus. $^{16}$

3. Wir betrachten nur die beiden Zeitpunkte 0 und $t$. Dennoch bietet sich die Intuition an, dass mit dem Heranreifen des Index ein Informationsprozeß verbunden ist. Entsprechend (5) würde man über die Jahre $1,2, \ldots, t$ immer mehr über die Verteilung von $\tilde{S}_{t}$ erfahren, weil im Verlauf der Zeit, sofern ein solcher betrachtet wird, die Realisationen Renditen (3), (4) bekannt werden. Insofern ist durch (5) eine gleichmäßige Form der "Risikoauflösung" gegeben.

\subsection{Beschreibung der Zahlung}

Wir wenden uns nun der Teilaufgabe zu, die eine Zahlung $\widetilde{Z}_{t}$ zu bewerten, das heißt, ihren Preis zum Zeitpunkt 0 im Finanzmarkt zu bestimmen. Aus der vierten Annahme folgt, dass die zu bewertende Zahlung lognormalverteilt ist. Um sie zu beschreiben, würde es genügen, das Niveau $a_{t}$ und die Steigung $b_{t}$ des Payoffs (5) zu bestimmen.

16 Wenn alle anderen Größen gleich bleiben, bewirkt eine Verkürzung der Reifezeit eine Erhöhung des Werts. 
Statt dessen könnte der Erwartungswert und die Standardabweichung von $\widetilde{Z}_{t}$ gegeben sein. Eine dritte Möglichkeit wäre, die zu bewertende Zahlung durch Quantile zu beschreiben, und auch dabei würden zwei "Punkte" genügen. Wir wählen diese dritte Möglichkeit der Beschreibung von $\widetilde{Z}_{t}$, weil sie praxisnah ist: Der Geschäftsplanung wird ein "besseres" und ein "schlechteres" Szenario entnommen. Dazu hilft beispielsweise ein Interview mit dem Experten, der die Informationen beschafft und $\widetilde{Z}_{t}$ als letztlich subjektive Wahrscheinlichkeitsverteilung aufstellt. Dem Experten wird gesagt: "Betrachten wir den Plan für das Jahr $t$. Um das Risiko des generierten Zahlungsüberschusses im Jahr $t$ genauer zu erfassen, wird eine Angabe benötigt, wie hoch er wäre, wenn sich die allgemeine Wirtschaft bis dahin gut entwickelt. In diesem besseren Szenario soll der Zahlungsüberschuß die mit $B_{t}$ bezeichnete Höhe haben. Zusätzlich wird eine Angabe benötigt, wie hoch die generierte Zahlung wäre, wenn sich die allgemeine Wirtschaft bis dahin schlecht entwickelt. Die Höhe der Zahlung in diesem schlechteren Szenario sei mit $A_{t}$ bezeichnet.

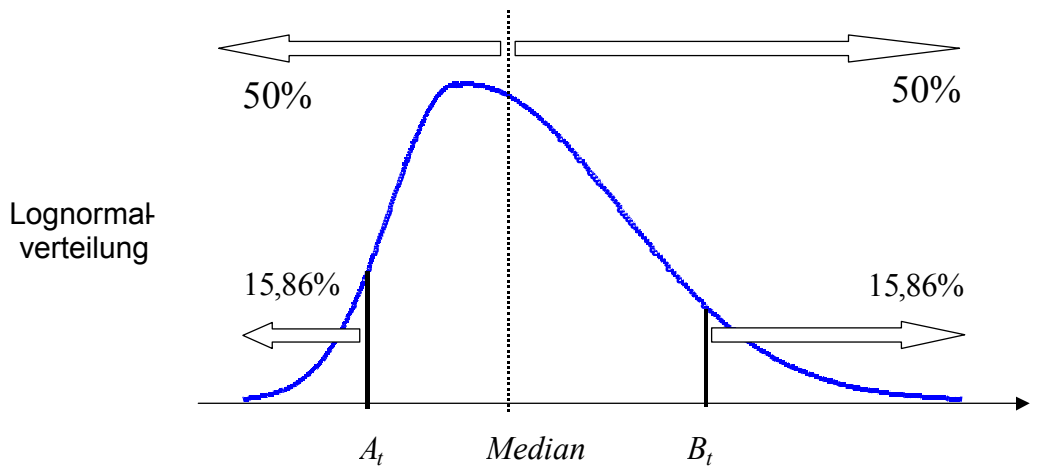

Figure 1: Es genügt, den Zahlungsüberschuß durch einen unteren und einen oberen Wert zu repräsentieren.

Die beiden Szenarien und die bei ihnen zu verzeichnenden Höhen des Zahlungsüberschusses $A_{t}$ und $B_{t}$ sollen sie als Quantile so bemessen sein, dass der Überschuß mit Wahrscheinlichkeit $1 / 6$, genauer $N(-1)=0,1586$, noch über der “Obergrenze" $B_{t}$ liegt. Mit derselben Wahrscheinlichkeit $1 / 6$ soll der Überschuß noch unterhalb der "Untergrenze" $A_{t}$ liegen. Aus dieser Beschreibung folgt, dass die Zahlung mit Wahrscheinlichkeit $2 / 3-$ genauer $N(1)-N(-1)=0,6827$ zwischen den beiden ermittelten Grenzen $A_{t}$ und $B_{t}$ liegt. Diese Informationsbeschaffung liefert eine Beschreibung der Zahlung $\widetilde{Z}_{t}$ durch zwei Realisationen: $A_{t}$ und $B_{t}$. Diese beiden "Punkte" spezifizieren die Abhängigkeit (5) vollständig. Hinweis: Der arithmetische Mittelwert $\left(A_{t}+B_{t}\right) / 2$ als "mittleres Szenario" von $\widetilde{Z}_{t}$ entspricht nicht genau dem Erwartungs- 
wert $E\left[\widetilde{Z}_{t}\right]$. Weil $\widetilde{Z}_{t}$ eine gewisse Rechtsschiefe aufweist, liegt das mittlere Szenario $\left(A_{t}+B_{t}\right) / 2$ etwas unterhalb des Erwartungswerts von $\tilde{Z}_{t}$.

\begin{tabular}{lcccccc}
\hline & $t=1$ & $t=2$ & 3 & 4 & 5 & $\cdots$ \\
\hline Obergrenze & $B_{1}$ & $B_{2}$ & $B_{3}$ & $B_{4}$ & $B_{5}$ & $\cdots$ \\
Untergrenze & $A_{1}$ & $A_{2}$ & $A_{3}$ & $A_{4}$ & $A_{5}$ & $\cdots$ \\
\hline
\end{tabular}

Figure 2: Der Geschäftsplan weist die Werte der Zahlungen für zwei Szenarien aus.

\subsection{Replikation}

Wir können nun fragen, welches Portfolio die durch zwei Szenarien beschriebene Zahlung nachbildet. Hierzu wählen wir ein Portfolio, das zum Zeitpunkt 0 den Geldbetrag $x$ verzinslich anlegt und parallel dazu den Geldbetrag $y$ in den Index investiert. Dieses Portfolio hat den Wert $x+y$. Wir bestimmen $x$ und $y$ so, dass es die zu bewertende Zahlung $\widetilde{Z}_{t}$ nachbildet - folglich gilt dann $W_{t}\left(\widetilde{Z}_{t}\right)=x+y$.

Notwendig und hinreichend für die Replikation ist, dass das Portfolio zum Zeitpunkt $t$ dieselbe Ober- und Untergrenze wie die Zahlung besitzt. Diese Bedingung lautet:

$$
\begin{aligned}
& B_{t}=x \cdot \exp (i \cdot t)+y \cdot \exp (\mu \cdot t+\sigma \cdot \sqrt{t}) \\
& A_{t}=x \cdot \exp (i \cdot t)+y \cdot \exp (\mu \cdot t-\sigma \cdot \sqrt{t})
\end{aligned}
$$

Denn die auf das Gesamtintervall von 0 bis $t$ bezogene stetige Rendite auf den Index - nochmals: sie ist normalverteilt - liegt im Sigma-Band, das von $\mu \cdot t-\sigma \cdot \sqrt{t}$ bis $\mu \cdot t+\sigma \cdot \sqrt{t}$ reicht. Im Gleichungssystem (6) sind $B_{t}$ und $A_{t}$ ebenso wie $t$ gegeben, auch die Finanzmarktdaten $i, \mu$ und $\sigma$ sind bekannt. Gesucht sind die Komponenten $x$ und $y$, die das Replikationsportfolio bestimmen. Um die Lösung einfacher herzuleiten, führen wir die Entwicklungsfaktoren

$$
u:=\exp (\mu \cdot t+\sigma \cdot \sqrt{t}), \quad d:=\exp (\mu \cdot t-\sigma \cdot \sqrt{t})
$$

für ein Up und ein Down des Index ein. Den Verzinsungsfaktor kürzen wir mit

$$
g=\exp (i \cdot t)
$$

ab. Es darf $d<g<u$ unterstellt werden. Das Gleichungssystem (6) hat mit diesen Bezeichnungen die Form 


$$
\begin{aligned}
& B_{t}=x \cdot g+y \cdot u \\
& A_{t}=x \cdot g+y \cdot d
\end{aligned}
$$

Leichte Umformungen führen auf die Lösung. Zieht man in (9) von der oberen die untere Gleichung ab, folgt $B_{t}-A_{t}=y \cdot(u-d)$ und somit $\left(B_{t}-A_{t}\right)((u-d)=y$. Multipliziert man die obere Gleichung mit $-d$, die untere mit $u$ und addiert sie, dann folgt $-d \cdot B_{t}+u \cdot A_{t}=x \cdot g \cdot(u-d)$ und damit $\left(-d \cdot B_{t}+u \cdot A_{t}\right) /(g \cdot(u-d))=x$. Zusammen lautet die gefundene Lösung

$$
x=\frac{u \cdot A_{t}-d \cdot B_{t}}{g \cdot(u-d)}, \quad y=\frac{-g \cdot A_{t}+g \cdot B_{t}}{g \cdot(u-d)}
$$

Der gesuchte Wert der durch die Schranken $A_{t}$ und $B_{t}$ beschriebenen und zum Zeitpunkt $t$ anfallenden unsicheren Zahlung ist die Summe $W_{t}\left(\widetilde{Z}_{t}\right)=x+y$, mithin:

$$
W\left(\tilde{Z}_{t}\right)=\left[\frac{u-g}{g \cdot(u-d)}\right] \cdot A_{t}+\left[\frac{g-d}{g \cdot(u-d)}\right] \cdot B_{t}
$$

Damit wären wir fertig: Der Wert der zu $t$ fälligen unsicheren Zahlung $\widetilde{Z}_{t}$, beschrieben durch ihre Höhen $B_{t}$ im guten Szenario (Marktentwicklung up) und $A_{t}$ im schlechten Szenario (Marktentwicklung down), ist bestimmt.

\subsection{Risikoneutrale Bewertung}

Die Wertformel (11) hat die Gestalt risikoneutraler Bewertung. Um das zu sehen, wird der Verzinsungsfaktor $g$ aus (11) herausgezogen. So erhält die Wertformel (11) die Form risikoneutraler Bewertung:

$$
W\left(\widetilde{Z}_{t}\right)=\frac{1}{g} \cdot\left\{(1-q) \cdot A_{t}+q \cdot B_{t}\right\}
$$

Die Pseudowahrscheinlichkeit (oder "risikoneutrale Wahrscheinlichkeit") für ein Up ist wie üblich mit $q$ bezeichnet,

$$
q:=\frac{g-d}{u-d}
$$

womit $1-q=u-g /(u-d)$ die Pseudowahrscheinlichkeit für ein Down ist. Beide Pseudowahrscheinlichkeiten liegen zwischen Null und Eins. Mit der risikoneutralen Bewertung ergibt sich diese Vorgehensweise: Zunächst wird der Pseudoerwartungswert aus Unterschranke $A_{t}$ und Oberschranke $B_{t}$ gebildet, $(1-q) \cdot A_{t}+q \cdot B_{t}$. Dieser Pseudoerwartungswert wird anschließend mit dem Zinssatz diskontiert. Insgesamt: 
1. Der Wert eines unsicheren und zu $t$ fälligen Zahlungsüberschusses $\widetilde{Z}_{t}$ ist der mit dem Zinssatz diskontierte Pseudoerwartungswert jener beiden Ergebnisse $A_{t}$ und $B_{t}$, die der Überschuß bei den beiden Ereignissen "schlechte" beziehungsweise "gute" Wirtschaftsentwicklung hat.

2. Die vorgeführte Replikation zeigte, dass sich der Wert in der Form risikoneutraler Bewertung darstellen läßt und lieferte die Pseudowahrscheinlichkeiten (13).

3. Die Diskontierung durch Replikation entspricht genau der Diskretisierung des Underlying, wie sie in einem Binomialmodell vorgenommen wird. Weil die zu bewertende Zahlung annahmegemäß einen linearen Payoff darstellt, genügt eine ganz einfache Diskretisierung: Das gesamte Zeitintervall von 0 bis $t$ wird als eine einzige Zeitstufe behandelt wird, in der nur ein Up und ein Down möglich sind.

4. Die Bewertungsformel (11) beziehungsweise (12) gilt allgemein für eine Zahlung, deren Payoff durch zwei Punkte beschreiben werden kann, und als Punkte wurden hier jene Realisationen des Underlying gewählt, auf die ein Up beziehungsweise ein Down führt. Ein besonderer Fall der Anwendung der Bewertung (11), (12) ist der, in dem die zu bewertende Zahlung bei beiden Szenarien übereinstimmt, $A_{t}=B_{t}$. In diesem Fall hat die $\mathrm{zu}$ bewertende Zahlung keine (systematische) Schwankungsbreite. Ihr Wert ist gleich der mit dem Zins diskontierten sicheren Zahlung, $W\left(\widetilde{Z}_{t}\right)=A_{t} / g=B_{t} / g$.

\subsection{Ein Zahlenbeispiel}

Dass mit (11) beziehungsweise (12) tatsächlich eine risikoneutrale Bewertung vorgenommen wird zeigt sich darin, dass die Daten der zu bewertenden Zahlung, also die Schranken $A_{t}$ und $B_{t}$ nicht in die Berechnung der Pseudowahrscheinlichkeiten einfließen: Das "Risiko" der Zahlung wird nicht vorweg benötigt, um die Pseudowahrscheinlichkeiten zu berechnen. Allein aufgrund der Finanzmarktdaten können die Pseudowahrscheinlichkeiten berechnet werden. Anschließend können die verschiedensten Zahlungen bewertet werden. Dies wird an einem Beispiel deutlich.

Beispiel 1: Gegeben sind $t=1$ und die Finanzmarktdaten $i=4 \%, \mu=7,61 \%$ und $\sigma=19,01 \%$. Daraus folgen nach (7) und (8) $u=1,3050, d=0,8923, g=1,0408$ und weiter nach (13) die Pseudowahrscheinlichkeiten $q=35,99 \%$ (für ein Up) und $1-q=64,01 \%$ (für ein Down). Nun können beliebige Zahlungen diskontiert werden, solange sie nur die Annahme 5 erfüllen (Lineare Abhängigkeit von der Wirtschaftsentwicklung, restliche Zufälligkeiten sind unsystematisch). Selbstverständlich müssen die Zahlungen durch ihre Schranken $A_{1}$ und $B_{1}$ gegeben sein. 
1. Im ersten Fall soll $A_{1}=100$ und $B_{1}=200$ sein. Der Wert ist gleich $(1 / 1,0408) \cdot(0,6401 \cdot 100+0,3599 \cdot 200)=130,66$.

2. Für $A_{1}=0$ und $B_{1}=100$ ist $(1 / 1,0408) \cdot(0,6401 \cdot 0+0,3599 \cdot 100)=34,58$ der Wert.

3. Für $A_{1}=-50$ und $B_{1}=50:(1 / 1,0408) \cdot(0,6401 \cdot(-50)+0,3599 \cdot 50)=-13,46$.

4. Für $A_{1}=-100$ und $B_{1}=0:(1 / 1,0408) \cdot(0,6401 \cdot(-100)+0,3599 \cdot 0)=-61,50$.

5. Für die durch $A_{1}=-200$ und $B_{1}=-100$ beschriebene Zahlung ist der Wert $(1 / 1,0408) \cdot(0,6401 \cdot(-200)+0,3599 \cdot(-100))=-157,58$.

Die Diskontierung durch Replikation kann auch Zahlungen bewerten, die einen sehr geringen oder einen negativen Erwartungswert aufweisen. Es ist möglich, dass eine Zahlung, die einen (geringen) positiven Erwartungswert besitzt, einen negativen Wert hat. Würde hier an der Risikoprämienmethode festgehalten werden, müßte die Diskontrate $r$ negativ gewählt werden. 1. Das entspricht nicht der Intuition. 2. Wenn die Korrelation mit dem Marktportfolio positiv ist, führt das CAPM auf ein positives Beta. Negative Diskontraten (bei positiver Korrelation mit dem Marktportfolio) lassen sich daher nicht mit dem CAPM erzeugen.

Die Diskontierung durch Replikation ist stets anwendbar, sofern der Finanzmarkt hinreichend viele Instrumente umfaßt - die bereits einen Preis haben - so dass die zu bewertende Zahlung in allen ihren relevanten Eigenschaften durch ein Portfolio aus diesen Instrumenten nachgebildet werden kann. Ist das geschehen, und der Markt arbitragefrei, so muss die zu bewertende Zahlung denselben Wert aufweisen wie das für die Replikation gebildete Portfolio.

\begin{tabular}{lllllllllll}
\hline $\boldsymbol{t}$ & $\mathbf{1}$ & $\mathbf{2}$ & $\mathbf{3}$ & $\mathbf{4}$ & $\mathbf{5}$ & $\mathbf{6}$ & $\mathbf{7}$ & $\mathbf{8}$ & $\mathbf{9}$ & $\mathbf{1 0}$ \\
\hline $\boldsymbol{q}$ & 35,99 & 30,52 & 26,53 & 23,34 & 20,68 & 18,39 & 16,39 & 14,63 & 13,07 & 11,66 \\
$\mathbf{1 - q}$ & 64,01 & 69,48 & 73,47 & 76,66 & 79,32 & 81,61 & 83,61 & 85,37 & 86,93 & 88,34 \\
\hline $\mathbf{1 / g}$ & 96,08 & 92,31 & 88,69 & 85,21 & 81,87 & 78,66 & 75,58 & 72,61 & 69,77 & 67,03 \\
\hline
\end{tabular}

Figure 3: Pseudowahrscheinlichkeiten für die Jahre 1 bis 10.

Beispiel 2: Gegeben sind $t=2$ und wieder die Finanzmarktdaten $i=4 \%, \mu=7,61 \%$, $\sigma=19,01 \%$. Daraus folgen mit (7) und (8) $u=1,5235, d=0,8899, g=1,0833$ und weiter die auch in der Tabelle (Figure 3) gezeigten Pseudowahrscheinlichkeiten $q=30,52 \%$ (Up) und $1-q=69,48 \%$ (Down). Nun können wieder beliebige Zahlungen diskontiert werden, solange sie nur die Annahme 5 erfüllen (Lineare Abhängigkeit von der Wirtschaftsentwicklung, restliche Zufälligkeiten sind unsystematisch) und durch ihre beiden Schranken $A_{2}$ und $B_{2}$ gegeben sind.

1. Für $A_{2}=0$ und $B_{2}=100$ ist der Wert $0,9231 \cdot(0,6948 \cdot 0+0,3052 \cdot 100)=28,17$. 
2. Für $A_{2}=-50, B_{2}=50$ ist der Wert $0,9231 \cdot(0,6948 \cdot(-50)+0,3052 \cdot 50)=-17,98$.

3. Für $A_{2}=-100, B_{2}=0$ ist der Wert $0,9231 \cdot(0,6948 \cdot(-100)+0,3052 \cdot 0)=-64,14$.

Um die Linearität der Wertfunktion zu illustrieren, weisen wir darauf hin, dass die Summe der ersten und der dritten Zahlung gerade das Doppelte der zweiten Zahlung ergibt. Entsprechend ist 28,17-64,14 = -35,97 das Doppelte von $-17,98$.

Eine Tabelle zeigt für den (stetigen) Zins $i=4 \%$ und die geschätzten Parameter der stetige Marktrendite $\mu=7,61 \%$ und $\sigma=19,01 \%$ die beiden Pseudowahrscheinlichkeiten $q$ (für ein Up) und $1-q$ (für ein Down) in Prozent. Außerdem ist der Diskontfaktor $1 / g=\exp (-i \cdot t)$ als Prozentzahl angegeben.

\section{$4 \quad$ Fallbeispiel}

\subsection{Daten}

Eine Unternehmung plant die Freien Cashflows im Detail für die kommenden drei Jahre. Der Planer nennt hierfür $100 \pm 30$, 140 \pm 50 , $200 \pm 70$. Der auf das Jahr 4 bezogene Fortführungswert wird ebenso prognostiziert, und zwar zu 2000 \pm 800 . Um die Spannbreite der möglichen Realisationen zu beschreiben, hat der Planer eine Bandbreite angegeben, so dass nur noch jeweils $1 / 3$ der sehr optimistischen Szenarien darüber und 1/3 der ganz pessimistischen Szenarien darunter liegen:

\begin{tabular}{lllll}
\hline & $t=1$ & $t=2$ & $t=3$ & $t=4$ \\
\hline Besseres Szenario & 130 & 190 & 270 & 2800 \\
Schlechteres Szenario & 70 & 90 & 130 & 1200 \\
\hline
\end{tabular}

Figure 4: Die Geschäftsplanung weist Cashflows für die Jahre 1 bis 3 sowie den auf das Jahr 4 bezogenen Fortführungswert durch jeweils zwei Szenarios aus.

Es soll mit den bisherigen Finanzmarktdaten $(i=4 \%, \mu=7,61 \%, \sigma=19,01 \%)$ gerechnet werden. Aufgrund dieser Angaben folgt für den Wert der Unternehmung: 


$$
\begin{aligned}
W= & 0,9608 \cdot(0,3599 \cdot 130+0,6401 \cdot 70)+0,9231 \cdot(0,3052 \cdot 190+0,6948 \cdot 90) \\
& +0,8869 \cdot(0,2653+0,7347 \cdot 130)+0,8521 \cdot(0,2334 \cdot 2800+0,7666 \cdot 1200)= \\
& 88,00+111,25+148,25+1340,85=1688,36
\end{aligned}
$$

Ein Anhänger traditioneller Diskontierung kann einwerfen, ob man dieses Ergebnis nicht auch mit der Risikoprämienmethode hätte finden können, in dem die "mittleren" Szenarien von 100 , 140, 200 für die Freien Cashflows und von 2000 für den Fortführungswert mit geeigneten Vergleichsrenditen diskontiert werden. Zur Antwort:

- Der Wert des ersten Freien Cashflows ist mit Replikation zu 88,00 berechnet und dieser Betrag läßt sich in der Form 100/(1+r $\left.r_{1}\right)$ der Risikoprämienmethode gewinnen, sofern $1+r_{1}=100 / 88,00=1,1364$ gewählt wird. Das heißt, die Diskontrate für das erste Jahr müßte $r_{1}=13,64 \%$ betragen. Achtung: Wir erwähnt ist das mittlere Szenario $\left(A_{t}+B_{t}\right) / 2$ nicht exakt der Erwartungswert, der bei der Risikoprämienmethode im Zähler steht. Wir fragen also, mit welcher Rendite des mittlere Szenario $\left(A_{t}+B_{t}\right) / 2$ zu diskontieren wäre. Diese Bemerkung gilt auch für die nachstehenden, illustrativen Rechnungen.

- Der Wert des zweiten Freien Cashflows ist 111,25. Er läßt sich in der Form $140 /\left(1+r_{2}\right)^{2}$ gewinnen, sofern $\left(1+r_{2}\right)^{2}=140 / 111,25=1,2584$ gewählt wird, das heißt, die Diskontrate - mit der das mittlere Szenario zu diskontieren wäre müßte für das zweite Jahr $r_{2}=12,18 \%$ betragen.

- Der Wert des dritten Freien Cashflows ist 148,25 und dieser Betrag läßt sich in der Form $200 /\left(1+r_{3}\right)^{3}$ gewinnen, sofern $\left(1+r_{3}\right)^{3}=200 / 148,25=1,3491$ gewählt wird. Die Diskontrate für das Jahr 3 müßte $r_{3}=10,50 \%$ betragen.

- Der Wert des Fortführungswerts ist 1340,85 und dieser Betrag läßt sich in der Form $2000 /\left(1+r_{4}\right)^{4}$ darstellen, sofern $\left(1+r_{4}\right)^{4}=2000 / 1340,85=1,4916$ gewählt wird, das heißt, die Diskontrate für das vierte Jahr müßte $r_{4}=10,51 \%$ betragen.

Zusammengefaßt:

$$
\begin{aligned}
& W=\frac{100}{1,1364}+\frac{140}{1,1218^{2}}+\frac{200}{1,1050^{3}}+\frac{2000}{1,1051^{4}}= \\
& =88,00+111,25+148,23+1340,98=1688,46
\end{aligned}
$$

Doch wie hätte man die richtigen Kapitalkosten für die Diskontierung nach der Risikoprämienmethode finden sollen? Noch ein Punkt: Man kann auch einen gleichförmigen Kapitalkostensatz $r$ finden, der 


$$
\frac{100}{1+r}+\frac{140}{(1+r)^{2}}+\frac{200}{(1+r)^{3}}+\frac{2000}{(1+r)^{4}}=1688,36
$$

bewirkt. Mit dem Excel-Solver ergibt sich

$$
r=10,62 \% \text {. }
$$

Doch wieder gilt: wie wäre man auf diese Kapitalkosten gekommen?

\subsection{Fortführung}

Der Planer hat bei der Aufstellung der Cashflows (Figure 4) vergessen, die Investitionen abzuziehen, wie das die DCF-Methode verlangt. Die Ausgaben für Investitionen stehen genau fest, es sind sichere Größen. Sie betragen in den ersten drei Jahren je 100 und der auf das vierte Jahr bezogene Barwert aller weiteren Investitionen ist 1000 . Die korrigierten Zahlungen sind: $100 \pm 30-100=0 \pm 30, \quad 140 \pm 50-100=40 \pm 50$, $200 \pm 70-100=100 \pm 70,2000 \pm 800-1000=1000 \pm 800$. Jemand hält fest: "Die Freien Cashflows werden im Sinne eines mittleren Szenarios mit 0, 40, 100 für die ersten drei Jahre geplant und für das vierte Jahr fließen 1000 als Fortführungswert in die Bewertung ein. Wenn diese mittleren Szenarien mit dem Kapitalkostensatz (15) gemäß der Risikoprämienmethode diskontiert werden, dann folgt:

$$
\frac{0}{1,1062}+\frac{40}{1,1062^{2}}+\frac{100}{1,1062^{3}}+\frac{1000}{1,1062^{4}}=774,39
$$

als Wert. Wir fragen, ob diese Rechnung stimmt. Die Bewertung mit Diskontierung durch Replikation geht nach Berücksichtigung der Investitionen von dem Plan aus:

\begin{tabular}{lccll}
\hline & $t=1$ & $t=2$ & $t=3$ & $t=4$ \\
\hline Besseres Szenario & 30 & 90 & 170 & 1800 \\
Schlechteres Szenario & -30 & -10 & 30 & 200
\end{tabular}

Figure 5: Der Plan mit Berücksichtigung der Investitionen.

Der korrekte, durch Replikation bestimmte Wert der Unternehmung ist:

$$
\begin{aligned}
W & =0,9608 \cdot(0,3599 \cdot 30+0,6401 \cdot(-30))+0,9231 \cdot(0,3052 \cdot 90+0,6948 \cdot(-10))+ \\
& +0,8869 \cdot(0,2653 \cdot 170+0,7347 \cdot 30)+0,8521 \cdot(0,2334 \cdot 1800+0,7666 \cdot 200)= \\
& =-8,08+18,94+59,55+488,71=559,13
\end{aligned}
$$

Zur Aufgabe, diesen korrekten Wert von 559,13 mit der Risikoprämienmethode (angewendet auf das mittlere Szenario) darzustellen: Der Wert des ersten Freien 
Cashflows ist mit Replikation zu $-8,08$ berechnet: Eine Position, die einen negativen Wert hat. Wer bei guter Wirtschaftsentwicklung 30 erhält aber bei schlechter Wirtschaftsentwicklung den selben Betrag nachlegen muss, der wird diese Position nur dann verpflichtend übernehmen, wenn man ihm 8,08 für das Underwriting gibt. Jedenfalls läßt sich dieser Wert $-8,08$ nicht in der Form $0 /\left(1+r_{1}\right)$ gewinnen, weil stets $0 /\left(1+r_{1}\right)=0$ gilt. Daraufhin betrachten wir die anderen Zeitpunkte nicht weiter. Immerhin ist möglich, einen gleichförmigen Kapitalkostensatz $r$ zu finden, der

$$
\frac{0}{1+r}+\frac{40}{(1+r)^{2}}+\frac{100}{(1+r)^{3}}+\frac{1000}{(1+r)^{4}}=559,13
$$

bewirkt. Mit dem Excel-Solver ergibt sich

$$
r=20,49 \% \text {. }
$$

Doch wie wäre man auf diesen Kapitalkostensatz gekommen und wie hätte man verstanden, dass er fast doppelt so hoch wie in (15) ist?

In einer zweiten Fortführung des Fallbeispiels ist der Planer erschrocken, weil er sich bei der Budgetierung der Investitionen gemäß Abschnitt 3.2 und Figure 5 vertan hatte. Die Ausgaben für die Investitionen betragen in den ersten drei Jahren nicht je 100 sondern je 200 und der auf das vierte Jahr bezogene Barwert aller weiteren Investitionen ist nicht 1000 sondern 2000. Die Freien Cashflows sind gemäß der neuerlichen Korrektur $100 \pm 30-200=-100 \pm 30 ， 140 \pm 50-200=-60 \pm 50,200 \pm 70-200=0 \pm 70$, $2000 \pm 800-2000=0 \pm 800$. Ein Anhänger traditioneller Diskontierung macht sich an die Korrektur und hält fest: “Die Freien Cashflows werden mit mittleren Szenarien von $-100,-60$, 0 für die ersten drei Jahre geplant und für Jahr 4 fließen 0 als Fortführungswert ein. Bei den Kapitalkosten ist unklar, ob sie nach (15) oder nach (19) zu bestimmen sind und es soll mit beiden Sätzen gerechnet werden. Deshalb sollte der Wert im Bereich

$$
\begin{aligned}
& \frac{-100}{1,1062}+\frac{-60}{1,1062^{2}}+\frac{0}{1,1062^{3}}+\frac{0}{1,1062^{4}}=-139,43 \\
& \frac{-100}{1,2049}+\frac{-60}{1,2049^{2}}+\frac{0}{1,2049^{3}}+\frac{0}{1,2049^{4}}=-124,32
\end{aligned}
$$

liegen. Das ist ein enger Bereich, weshalb der Wert wohl ziemlich genau bestimmt ist." Um sicher zu gehen, soll mit Replikation diskontiert werden. Die beiden Szenarien der Freien Cashflows sind:

\begin{tabular}{lllll}
\hline & $t=1$ & $t=2$ & $t=3$ & $t=4$ \\
\hline Besseres Szenario & -70 & -10 & 70 & 800 \\
Schlechteres Szenario & -130 & -110 & -70 & -800 \\
\hline
\end{tabular}


Es wird mit Tabelle (Figure 3) bewertet:

$$
\begin{aligned}
W & = \\
& 0,9608 \cdot(0,3599 \cdot(-70)+0,6401 \cdot(-130))+0,9231 \cdot(0,3052 \cdot(-10)+0,6948 \cdot(-110))+ \\
& +0,8869 \cdot(0,2653 \cdot 70+0,7347 \cdot(-70))+0,8521 \cdot(0,2334 \cdot 800+0,7666 \cdot(-800)= \\
& =-104,15-73,37-29,14-363,43=-570,10
\end{aligned}
$$

Der große Unterschied zwischen dem durch Replikation gefundenen Wert (21) und den Rechnungen nach der Risikoprämienmethode (20) überrascht. Wir überlegen, ob der korrekte Wert (21) auch mit der "simple discounting rule" hätte gefunden werden können. Der Ansatz lautet

$$
\frac{-100}{1+r}+\frac{-60}{(1+r)^{2}}+\frac{0}{(1+r)^{3}}+\frac{0}{(1+r)^{4}}=-570,10 .
$$

Für $(1+r)>1$ ist diese Gleichung nicht erfüllbar; die Diskontrate müßte negativ sein. Sie müßte $r=-57,62 \%$ betragen, damit eine Diskontierung mit ihr den korrekten Wert liefert.

\subsection{Ergebnisse}

1. Die vorgeführte Diskontierung (Wertfindung einer in Zukunft fälligen Zahlung) durch Replikation setzt zwar voraus, dass die zu $t$ fällige und zu bewertende unsichere Zahlung durch eine Untergrenze $A_{t}$ und eine Obergrenze $B_{t}$ beschrieben wird. Indessen sind keine Angaben über ein Beta verlangt. Im Unterschied dazu verlangt die Risikoprämienmethode die Kenntnis der Diskontrate.

2. Bei der Diskontierung durch Replikation wirken sich die unteren Realisationen der zu bewertenden Zahlung besonders stark und abträglich auf den Wert aus: Die Tabelle (Figure 3) zeigt, dass die Pseudowahrscheinlichkeiten für das Down stets größer als die für das Up sind (obwohl beide Entwicklungen dieselbe objektive Wahrscheinlichkeit haben). Das starke Gewicht der Untergrenze $A$ der Zahlung ist besonders hoch, wenn der Fälligkeitszeitpunkt $t$ spät ist. Das bedeutet: Für großes $t$ ist der Wert praktisch allein durch $A_{t}$ bestimmt, die Höhe der Zahlung, die sie bei einer schlechten Wirtschaftsentwicklung hat.

3. Besonders wenn die Untergrenze $A_{t}$ der Cashflows gering ist, wird der Wert durch die Risikoprämienmethode bei Verwendung "üblicher" Diskontsätze als zu hoch eingeschätzt; der korrekte (durch Diskontierung mit Replikation gefundene) Wert ist in solchen Situationen geringer. Unter Umständen ist der Wert der Zahlung sogar negativ. Das ist zu beachten, wenn die Unternehmung sich in einer Phase der Restrukturierung befindet und besonders in den ersten Jahren die Freien Cashflows 
negativ sind. Hier kann die Risikoprämienmethode leicht zu Ergebnissen führen, die den korrekten Wert überschätzen. Eine Firma in der Restrukturierung, bei der sehr geringe oder sogar negative Freie Cashflows eintreten oder eintreten könnten, hat einen geringeren Wert als mit der Risikoprämienmethode bei "typischen Kapitalkostensätzen" ermittelt wird.

4. Wird dennoch an der Risikoprämienmethode festgehalten, so zeigt sich, dass gelegentlich nur negative Diskontraten den korrekten Wert erzeugen. Negative Diskontraten widersprechen der Intuition. Auch gibt es einen Widerspruch zum CAPM insofern, als aufgrund der positiven Korrelation der Zahlung mit dem Marktindex das Beta positiv sein sollte. Positive Betas führen auf Diskontsätze, die den Zinssatz übersteigen. Zudem gilt auch hier, dass man ihre Höhe nur nachträglich ermitteln kann, wenn zuvor der Wert (mit Replikation) bestimmt wurde.

Literatur:

WOLFGANG BALLWIESER (1990): Unternehmensbewertung und Komplexitätsreduktion. 3. Auflage, Gabler, Wiesbaden.

FISHER BLACK (1988): A Simple Discounting Rule. Financial Management, pp. 7-11.

MiCHAEL BRENNAN (1973): An Approach to the Valuation of Uncertain Income Streams. Journal of Finance 28, pp. 661-674.

Michael BRENNAN: The Pricing of Contingent Claims in Discrete Time Models. Journal of Finance 34 (1979), pp. 91-102.

GEORGE CONSTANTINIDES (1988): Theory of Valuation - Overview and Recent Developments, in BHATTACHARYA und CONSTANTINIDES (Ed.), Theory of Valuation - Frontiers of Modern Financial Theory, Volume 1, pp. 1-23.

HAROLD DEMSETZ: The structure of ownership and the theory of the firm. Journal of Law and Economics 26 (1983), pp. 375-390.

EUGENE F. FAMA (1977): Risk-Adjusted Discount Rates And Capital Budgeting Under Uncertainty. Journal of Financial Economics 5, pp. 3-24.

EUGENE F. FAMA und MiCHAEL JENSEN: Separation of ownership and control. Journal of Law and Economics 26 (1983), pp, 301-325.

J. MiCHAEL HARRISON und DAVID M. KREPS (1979): Martingales and arbitrage in multiperiod securities markets. Journal of Economic Theory 20, pp. 381-408.

JOHN R. HICKS: Value and Capital. Oxford 1939.

G. Hubermann: A Simple Approach to Arbitrage Pricing Theory. Journal of Economic Theory 28 (1982), pp. 183-191.

LUTZ KRUSCHWITZ und A. LÖFFLER: Unendliche Probleme bei der Unternehemnsbewertung. Die Betriebswirtschaft 51, pp. 1041-1043.

LUTZ KRUSCHWITZ (2002): Finanzierung und Investition, 3. Auflage, Oldenbourg, München.

WOLFGANG KÜRSTEN (2002): 'Unternehmensbewertung unter Unsicherheit' oder: Theoriedefizit einer künstlichen Diskussion über Sicherheitsäquivalent- und Risikozuschlagsmethode Anmerkungen (nicht nur) zu dem Beitrag von Bernhard Schwetzler in der zfbf. Zeitschrift für betriebswirtschaftliche Forschung 54, pp. 128-144.

Michael JenSEN und William H. MECKLING: Theory of the firm: Managerial behavior, agency costs and ownership structure. Journal of Financial Economics 3 (1976), pp. 305-360.

JÖRG LAITENBERGER: Rendite und Kapitalkosten. Working Paper Lehrstuhl Banken und Finanzierung der Universität Hannover, 21.04.2004.

ROBERT C. MERTON (1973): Theory of Rational Option Pricing, wieder abgedruckt in MERTON, Continuous Finance, Kapitel 8. 
FRANK RICHTER (2001): Simplified Discounting Rules in Binomial Models. Schmalenbach Business Review 53, pp. 175-196.

STEVEN Ross (1978): A Simple Approach to the Valuation of Risky Streams. Journal of Business 51, pp. $453-475$.

MARK RUBINSTEIN (1976): The Valuation of Uncertain Income Streams and the Pricing of Options. Bell Journal of Economics 1, pp. 225-244.

BERNHARD SCHWETZLER (2000): Unternehmensbewertung unter Unsicherheit - Sicherheitsäquivalent- oder Risikozuschlagsmethode? zfbf - Schmalenbachs Zeitschrift für betriebswirtschaftliche Forschung 52, Heft 5, pp. 469-486.

KlaUS SPREMANN (1986): The Simple Analytics of Arbitrage, in: Günter Bamberg and Klaus Spremann (eds.), Capital Market Equilibria, Springer-Verlag, Berlin 1986, pp. 189-207.

KLAUS SPREMANN (2002): Finanzanalyse und Unternehmensbewertung. Oldenbourg, München; 2. Auflage unter dem Titel Modern Finance - Rendite, Risiko, Wert, 2004.

KLAUS SPREMANN (2004): Valuation - Grundlagen moderner Unternehemnsbewertung. Oldenbourg, München 2004.

WOLFGANG STÜTZEL: Preis, Wert und Macht. Dissertation Tübingen 1952 (Neudruck Aalen 1972).

JOCHEN WILHELM (2002): Risikoabschläge, Risikozuschläge und Risikoprämien: Finanzierungstheoretische Anmerkungen zu einem Grundproblem der Unternehmensbewertung. Working Paper, Universität Passau, Wirtschaftswissenschaftliche Fakultät.

WALDEMAR WITTMANN: Der Wertbegriff in der Betriebswirtschaftslehre. Köln und Opladen 1956. 